\title{
Smarte Sensorik für industrielle Anwendungen
}

\author{
T. Sauter OVE
}

Online publiziert am 12. September 2018

(c) Springer-Verlag GmbH Austria, ein Teil von Springer Nature 2018

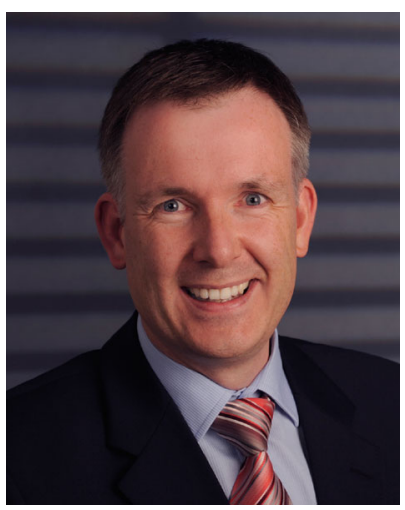

Thilo Sauter

\section{Sehr geehrte Leserinnen} und Leser,

Sensoren sind aus unserem Leben nicht mehr wegzudenken. Wir haben uns daran gewöhnt, dass unsere Autos voller Sensorelektronik stecken, dass das Telefonieren bei unseren Mobiltelefonen gegenüber Schrittzählen, Pulsmessen und Positionsbestimmung fast ein nebensächliches Feature ist, und dass selbst unsere Zahnbürsten smart werden. Doch nicht nur in

Dingen des täglichen Bedarfs finden sich mehr und mehr Sensoren. Unverzichtbar sind sie in der Automatisierungstechnik, wo sie Fertigungsprozesse steuern oder die Qualität von Produkten überwachen. Dabei lassen sich die Sensoren in solchen Anwendungsgebieten immer weniger als isolierte Elemente betrachten. Steigende Anforderungen an Robustheit, Genauigkeit und Preis zwingen da$z u$, die messtechnische Aufgabe ganzheitlich zu sehen und letztlich ein Sensorsystem zu entwerfen, das für die jeweilige Anwendung optimiert ist.

Diese Überlegungen waren der Ausgangspunkt für die Österreichische Gesellschaft für Mess-, Automatisierungs- und Robotertechnik (GMAR), das vorliegende Heft der Sensorik für industrielle Anwendungen zu widmen. Es illustriert anhand einiger Beispiele, wie vielfältig und praxisbezogen die österreichische Forschungslandschaft im Bereich Messtechnik und Sensorik ist und wie gut die Kooperation zwischen Wissenschaft und Industrie funktioniert. Nicht zuletzt zeigen die nachfolgend kurz beschriebenen Beiträge auch, wie eng verzahnt die Teilbereiche der GMAR sind.

Csencsics et al. beschäftigen sich mit roboterbasierter 3D-InProzess Messtechnik. Für flexible Fertigungssysteme müssen auch die verwendeten Messsysteme flexibel sein, wofür Roboter als Träger der Sensoren denkbar gut geeignet sind. Die exakte Positionierung zwischen robotergeführtem Sensor und Werkstück ist dabei eine große Herausforderung, speziell wenn es darum geht, mithilfe des Roboters dreidimensional zu messen. Der Beitrag stellt einen integrierten Systementwurf vor, der auf die Verwendung von optischen Sensoren zugeschnitten ist.

Der Beitrag von Exel et al. ist der berührungslosen Temperaturmessung in rauen industriellen Umgebungen gewidmet, die trotz scheinbarer Einfachheit ein komplexes messtechnisches Problem darstellt. Während es für die Messung von Oberflächentemperaturen etablierte Verfahren gibt, stellt die Messung von Gastemperaturen nach wie vor eine Herausforderung dar. Der Beitrag unter- sucht die aus der Meteorologie bekannte Methode der DopplerRadar-Messung der Schallgeschwindigkeit, die temperaturabhängig ist und bei geeigneter Ausführung der Wandler auch ortsaufgelöst möglich ist. Damit wird die Bestimmung von Temperaturprofilen in industriellen Prozessgasen möglich.

Bibl et al. stellen in ihrem Beitrag einen optischen Sensor vor, der seine Lage relativ zu einer fixen Laserreferenz bestimmen kann. Das Ziel der Arbeit ist letztlich, sich autonom bewegende Geräte entlang des Lasers präzise im Bereich von Zehntelmillimetern auszurichten. Das einleuchtende Anwendungsbeispiel dafür sind Maschinen, die bei der Errichtung von Gebäuden Fußböden automatisiert herstellen und nivellieren können.

Der Beitrag von Enser et al. widmet sich dem hochaktuellen Thema von gedruckten Sensoren, die in Polymerbeschichtungen eingebettet werden können. Solche Sensoren können kostengünstig und ohne weitere Vorbehandlung auf unterschiedliche Trägermaterialien aufgebracht werden, wobei allerdings die Rauheit der Oberfläche eine Herausforderung an die Fertigung und Genauigkeit des Sensors darstellt. Der Beitrag zeigt dies am Beispiel von kapazitiven, Dehnungs- und piezo-/pyroelektrischen Sensoren auf Metallblechen.

Yoo et al. beschäftigen sich mit Sensorik für das autonome Fahren, konkret mit Lidar-Sensoren, dem lichtbasierten Pendant zu Radar-Sensoren. Diese wären grundsätzlich in der Lage, genaue 3DBilder der Umgebung des $\mathrm{Kfz}$ bei unterschiedlichsten Wetterbedingungen zu liefern, sind aber derzeit preislich und auflösungsmäßig noch nicht attraktiv genug. Der Beitrag diskutiert Lidar-Sensoren, die mit Hilfe von mikromechanischen Spiegeln die Umgebung abtasten können und im Vergleich zu anderen Scannern sowohl kostengünstig als auch kompakt hergestellt werden können.

Einen anderen Systemaspekt greifen Knoll et al. in ihrem Beitrag auf: die Bedeutung von drahtlosen Netzwerken für verteilte Datenerfassung. Smarte, vernetzte Sensorsysteme sind besonders dann von Vorteil, wenn die Überwachung räumlich verteilter Größen gefordert ist. Der Beitrag zeigt dies am Beispiel von Luftgütemessungen, die traditionell aus Kostengründen mit wenigen Messgeräten auskommen müssen. Eine feinere örtliche Auflösung mit kostengünstigeren Sensoren kann einen besseren Überblick über die tatsächliche Luftgüte in einem Gebiet geben. Dafür sind aber, neben den Sensoren, auch effiziente Netzwerke nötig, die eine großräumige Vernetzung ermöglichen.

Mein Dank gilt den Autoren für ihre breit gefächerten Beiträge und den Gutachtern, die mit ihren konstruktiven Kommentaren geholfen haben, die Beiträge noch informativer zu machen. Ich hoffe, sehr geehrte Leserinnen und Leser, dass Ihnen das vorliegende Heft einen interessanten Einblick in die vielfältige Welt der Sensorik und Messtechnik und ihrer Anwendungen gibt, und wünsche eine spannende und anregende Lektüre.

Sauter, Thilo, Institut für Computertechnik, Technische Universität Wien, Gußhausstraße 27-29, 1040 Wien, Österreich (E-Mail: thilo.sauter@tuwien.ac.at) 